\title{
The roots of resistance
}

\section{Learning how melanoma fights back may yield new therapies.}

\section{BY HEIDI LEDFORD}

A promising new cancer drug called PLX4032 made waves earlier this year for its success against the deadly cancer melanoma. The results of a clinical trial were dramatic, with some patients emerging nearly cancer-free. But for most, the drug stopped working about seven months after therapy began, and the tumours returned ${ }^{1}$. This week, two follow-up studies reveal why PLX4032 fails and how tumours' resistance to it might be overcome.

About half of patients with melanoma have a mutated version of the B-RAF protein, which is thought to help trigger the growth of tumour cells. PLX4032 was designed by Plexxikon, a pharmaceutical company in Berkeley, California, to selectively inhibit it. In one study ${ }^{2}$, PLX4032 shrank tumours in 24 of 32 patients with B-RAF mutations. But tumours often evolve ways to evade treatment, and this was no exception. Levi Garraway, an oncologist at the Dana-Farber
Cancer Institute in Boston, Massachusetts, and his colleagues have now found that excessive production of a cancer-promoting protein called COT can shield cultured cells from PLX4032. High levels of COT were also found in two of three PLX4032-resistant tumours taken from patients who had received the drug ${ }^{3}$.

A group led by Roger Lo, a dermatologist at the University of California, Los Angeles, found two other ways for tumour cells to short-circuit PLX4032: by reactivating the B-RAF signalling pathway damped down by the drug, or by stimulating tumour-cell growth mediated by a different protein ${ }^{4}$. Larger studies will be needed to assess the importance of both groups' results for clinical applications.

Still, the findings are a crucial step towards determining how to overcome PLX4032 resistance, says Alexis Borisy, chief executive of Foundation Medicine in Cambridge, Massachusetts, which is developing diagnostic tests for tumours. Pharmaceutical firms have already started clinical trials to test PLX4032 in combination with experimental drugs that inhibit MEK, a protein involved in cell growth. When Garraway's team tested this combination in their cultured tumour cells, they found that it overcame the effects of high levels of COT expression.

Clinicians may need to combine four or more drugs to create a potent cancer-fighting cocktail, says Borisy. PLX4032 is a good place to start, he notes: its highly selective targeting of B-RAF means it could have fewer unwanted side effects. "It's an exciting opportunity," he says.

1. Ledford, H. Nature 467, 140-141 (2010).

2. Flaherty, K. T. et al. N. Engl. J. Med. 363, 809-819 (2010).

3. Johannessen, C. M. et al. Nature advance online publication doi:10.1038/nature09627 (2010).

4. Nazarian, R. et al. Nature advance online publication doi:10.1038/nature09626 (2010).

\section{CORRECTION}

The News story 'China tackles surge in mental illness' (Nature 468, 145; 2010) incorrectly implied that Wanjun Guo leads a 20-million-yuan project to survey mental disorders in the "post Wenchuan earthquake' region. Guo is merely a participant in the project, which is itself only part of a 20-million-yuan effort to study the psychological impact of the quake and identify potential interventions. 